\title{
Observation error statistics for Doppler radar radial wind superobservations assimilated into the DWD COSMO-KENDA system
}

Article

Published Version

Open Access

Waller, J. A., Bauernschubert, E., Dance, S. L., Nichols, N. K., Potthast, R. and Simonin, D. (2019) Observation error statistics for Doppler radar radial wind superobservations assimilated into the DWD COSMO-KENDA system. Monthly Weather Review, 147. pp. 3351-3364. ISSN 0027-0644 doi: https://doi.org/10.1175/MWR-D-19-0104.1 Available at https://centaur.reading.ac.uk/84712/

It is advisable to refer to the publisher's version if you intend to cite from the work. See Guidance on citing.

To link to this article DOI: http://dx.doi.org/10.1175/MWR-D-19-0104.1

Publisher: American Meteorological Society

All outputs in CentAUR are protected by Intellectual Property Rights law, including copyright law. Copyright and IPR is retained by the creators or other copyright holders. Terms and conditions for use of this material are defined in the End User Agreement. 


\section{www.reading.ac.uk/centaur}

\section{CentAUR}

Central Archive at the University of Reading

Reading's research outputs online 


\title{
• Observation Error Statistics for Doppler Radar Radial Wind Superobservations Assimilated into the DWD COSMO-KENDA System
}

\author{
J. A. WALLER \\ School of Mathematical, Physical and Computational Sciences, University of Reading, \\ Reading, United Kingdom \\ E. BAUERNSCHUBERT \\ Deutscher Wetterdienst, Offenbach, Germany \\ S. L. DANCE AND N. K. NiCHOLS \\ School of Mathematical, Physical and Computational Sciences, University of Reading, Reading, United Kingdom \\ R. POTTHAST \\ School of Mathematical, Physical and Computational Sciences, University of Reading, Reading, \\ United Kingdom, and Deutscher Wetterdienst, Offenbach, Germany \\ D. SIMONIN \\ MetOffice@Reading, University of Reading, Reading, United Kingdom
}

(Manuscript received 9 April 2019, in final form 19 June 2019)

\begin{abstract}
Currently in operational numerical weather prediction (NWP) the density of high-resolution observations, such as Doppler radar radial winds (DRWs), is severely reduced in part to avoid violating the assumption of uncorrelated observation errors. To improve the quantity of observations used and the impact that they have on the forecast requires an accurate specification of the observation uncertainties. Observation uncertainties can be estimated using a simple diagnostic that utilizes the statistical averages of observation-minusbackground and observation-minus-analysis residuals. We are the first to use a modified form of the diagnostic to estimate spatial correlations for observations used in an operational ensemble data assimilation system. The uncertainties for DRW superobservations assimilated into the Deutscher Wetterdienst convectionpermitting NWP model are estimated and compared to previous uncertainty estimates for DRWs. The new results show that most diagnosed standard deviations are smaller than those used in the assimilation, hence, it may be feasible to assimilate DRWs using reduced error standard deviations. However, some of the estimated standard deviations are considerably larger than those used in the assimilation; these large errors highlight areas where the observation processing system may be improved. The error correlation length scales are larger than the observation separation distance and influenced by both the superobbing procedure and observation operator. This is supported by comparing these results to our previous study using Met Office data. Our results suggest that DRW error correlations may be reduced by improving the superobbing procedure and observation operator; however, any remaining correlations should be accounted for in the assimilation.
\end{abstract}

¿ Denotes content that is immediately available upon publication as open access.

Corresponding author: J. A. Waller, j.a.waller@reading.ac.uk

\section{Introduction}

It is now routine for national weather prediction centers to run a convection-permitting forecast model with resolutions of $1.5-3 \mathrm{~km}$ (Baldauf et al. 2011; Tang et al. 2013; Müller et al. 2017). At the convective scale the errors in the background are highly flow dependent (Chung et al. 2013) and, hence, it is beneficial to determine 
the background error covariance matrix explicitly using ensemble data assimilation. The local ensemble transform Kalman filter (LETKF) (Hunt et al. 2007) is an efficient ensemble data assimilation scheme that is suitable for use in numerical weather prediction (NWP), and the Deutscher Wetterdienst (DWD) are now using it for operational data assimilation (Schraff et al. 2016). To further advance the quality of convection-permitting NWP it is important to assimilate observations containing detailed information on appropriate scales (Sun et al. 2014; Clark et al. 2016; Ballard et al. 2016; Gustafsson et al. 2018). One such set of observations are Doppler radar radial winds (DRWs). The assimilation of DRWs provides a significant positive impact on the forecast (Montmerle and Faccani 2009; Xue et al. 2013, 2014) and as a result they are now assimilated at a number of operational centers (Xiao et al. 2008; Simonin et al. 2014). However, to effectively assimilate high-resolution observations it is necessary to understand and correctly account for their error statistics (Gorin and Tsyrulnikov 2011).

In data assimilation, the observation errors contain contributions from both the instrument error and representation error (Janjić et al. 2018). It has been shown that the observation errors may be correlated, state dependent and, due to the mismatch in scales represented by the model and the observation, dependent on the model resolution (Waller et al. 2014; Hodyss and Nichols 2015). Yet, until recently, observation errors have been assumed uncorrelated and the data are often thinned or "superobbed" (Lorenc 1981) in an attempt to satisfy this assumption. However, the inclusion of satellite interchannel error correlations in data assimilation systems has been shown to lead to improvements in the forecast skill score (Weston et al. 2014; Bormann et al. 2016; Campbell et al. 2017). Furthermore, the inclusion of spatially correlated DRW errors in the assimilation permits an increased percentage of high-resolution DRW observations to be assimilated (Simonin et al. 2019). Theoretical work has also shown that improved specification of observation error statistics in data assimilation can improve analysis accuracy and allow the inclusion of more observation information content (Stewart et al. 2008, 2013; Fowler et al. 2018).

Observation uncertainties must be estimated statistically. Desroziers et al. (2005) proposed a method that has become popular for estimating observation error statistics due to its simplicity. The diagnostic provides an estimate of the observation error covariance matrix using the statistical average of observation-minus-background and observation-minus-analysis residuals. A detailed discussion of this diagnostic and its limitations is given in section 2. Despite these limitations the diagnostic has been successfully used in operational settings to estimate satellite interchannel error covariances (Stewart et al. 2014; Weston et al. 2014; Bormann et al. 2016), temporal error covariances (Bennitt et al. 2017) and spatial error covariances (Waller et al. 2016a; Cordoba et al. 2017). Spatial error correlations for DRWs have previously been calculated for observations assimilated at the Met Office and Météo-France (Waller et al. 2016c; Wattrelot et al. 2012). The diagnostics can also be used to learn about the assimilation system. For example, to test selfconsistency in the system (Desroziers et al. 2005), check for observation bias (Waller et al. 2016a) and to determine sources of errors (Waller et al. 2016c) as well as providing guidance for quality control procedures (Waller et al. 2018).

In this manuscript we use the diagnostic to estimate observation error statistics for DRW superobservations (DRWSOs) assimilated into the convection-permitting model used at DWD. To gain insight into how different observation processing systems affect observation error statistics, we compare our estimated uncertainties to uncertainties estimated for DRWs assimilated at the Met Office. The comparison between these two estimated statistics also allows us to consider how the use of a flow dependent background error covariance matrix impacts the results.

A review of the diagnostic method is given in section 2 . In section 3 we describe the NWP model, assimilation system and the DRWSOs used at DWD. In section 4 we describe the experimental design used when calculating the average horizontal error statistics for DRWSOs. We describe our new results in section 5 . We find that the majority of the estimated error standard deviations for the DRWSOs assimilated into the COSMO-DE model are smaller than those used in the assimilation suggesting that it may be feasible to reduce the DRWSO error standard deviations in the assimilation scheme. However, for observations above $10 \mathrm{~km}$, or those from highelevation beams, the estimated standard deviations are considerably larger than those used in the assimilation. Comparisons between different radars highlight that contaminated observations have been assimilated and the quality control procedure needs to be improved. The correlation length scales are longer than the observation separation distance.

In section $5 c$ the averaged horizontal error statistics are compared to the observation error statistics for DRWSOs assimilated in the Met Office convectionpermitting model calculated in Waller et al. (2016c). There are a number of similarities between the results; these support the hypothesis that simplifications in the observation operator cause some correlation between observation errors. Finally we conclude in section 6 . 


\section{The diagnostic of Desroziers et al. (2005)}

Following Waller et al. (2016c) we describe data assimilation and the diagnostic proposed in Desroziers et al. (2005).

\section{a. Data assimilation}

Data assimilation techniques combine a model prediction of the state, the background $\mathbf{x}^{b} \in \mathbb{R}^{N^{m}}$, with observations $\mathbf{y} \in \mathbb{R}^{N^{p}}$, to provide a best estimate of the state, $\mathbf{x}^{a} \in \mathbb{R}^{N^{m}}$, known as the analysis. In the assimilation the observations and background are weighted by their respective uncertainties using the background and observation error covariance matrices $\mathbf{B} \in \mathbb{R}^{N^{m} \times N^{m}}$ and $\mathbf{R} \in \mathbb{R}^{N^{p} \times N^{p}}$. To calculate the analysis the background must be mapped into the observation space using the possibly nonlinear observation operator, $\mathscr{H}: \mathbb{R}^{N^{m}} \rightarrow \mathbb{R}^{N^{p}}$.

\section{b. The diagnostic}

Desroziers et al. (2005) show that the observation error covariance matrix can be estimated using the observation-minus-background and observation-minusanalysis residuals. The background residual, also known as the innovation,

$$
\mathbf{d}_{b}^{o}=\mathbf{y}-\mathscr{H}\left(\mathbf{x}^{b}\right),
$$

is the difference between the observation $\mathbf{y}$ and the mapping of the forecast vector $\mathbf{x}^{b}$ into observation space by the observation operator $\mathscr{H}$. The analysis residual,

$$
\begin{aligned}
\mathbf{d}_{a}^{o} & =\mathbf{y}-\mathscr{H}\left(\mathbf{x}^{a}\right), \\
& \approx \mathbf{y}-\mathscr{H}\left(\mathbf{x}^{b}\right)-\mathbf{H K} \mathbf{d}_{b}^{o},
\end{aligned}
$$

is similar to the background residual, but with the forecast vector replaced by the analysis vector $\mathbf{x}^{a}$. The analysis is determined using

$$
\mathbf{x}^{a}=\mathbf{x}^{b}+\mathbf{K}\left[\mathbf{y}-\mathscr{H}\left(\mathbf{x}^{b}\right)\right],
$$

where $\mathbf{K}=\mathbf{B} \mathbf{H}^{\mathrm{T}}\left(\mathbf{H B H} \mathbf{H}^{\mathrm{T}}+\mathbf{R}\right)^{-1}$ is the gain matrix and $\mathbf{H}$ is the linearized observation operator, linearized about the current state. Taking the statistical expectation of the product of the analysis and background residuals, and assuming that the forecast and observation errors are uncorrelated, results in

$$
E\left[\mathbf{d}_{a}^{o} \mathbf{d}_{b}^{o \mathrm{~T}}\right] \approx \mathbf{R} .
$$

Due to the statistical nature of the diagnostic the resulting matrix will not be symmetric. Therefore, if the matrix is to be used it must be symmetrized.

\section{c. The diagnostic for local assimilation}

The diagnostic in Desroziers et al. (2005) is derived assuming that the analysis is calculated using minimumvariance linear statistical estimation. However, the diagnostic no longer provides a correct estimate of the observation error covariance matrix if local ensemble data assimilation is used to calculate the analysis. Using a modified version of the diagnostic it is possible to recover some of the observation error statistics. Waller et al. (2017) show that the diagnostic can be used to estimate the error correlations between two observations if the observation operator that determines the model equivalent of observation $\mathbf{y}_{i}$ acts only on states that have been updated using the observation $\mathbf{y}_{j}$. Otherwise, the error correlation cannot be estimated using the diagnostic.

\section{d. Limitations of the diagnostic}

A correct estimate of the observation error covariance matrix is given by the diagnostic in Eq. (5) only if the background and observation error statistics used in assimilation exactly represent the true statistics. However, successive iterations of the diagnostic may improve the result (Desroziers et al. 2005). Furthermore, when the assumed statistics used in the assimilation are not exact, with careful interpretation of the results, the diagnostic can still provide useful information about the true observation uncertainties (Waller et al. 2016b; Ménard 2016). The diagnostic has further limitations; these include the assumption that the observation operator is linear (Terasaki and Miyoshi 2014) and the fact that ergodic, isotropic and homogeneous assumptions are often made in order to obtain sufficient sample residuals (Todling 2015). Because of the limitations of the diagnostic, observation error statistics estimated using this methodology should be interpreted as indicative, rather than necessarily quantitatively perfect.

\section{DWD model, assimilation, and observations}

\section{a. The operational COSMO-KENDA system}

The COSMO (Consortium for Small-Scale Modeling in Numerical Weather Prediction) model is a limitedarea NWP model developed and maintained by the national weather services of the COSMO consortium. At the DWD the COSMO-DE version of the model, covering Germany and parts of the neighboring countries, is run operationally (Baldauf et al. 2011). The COSMO-DE model has a horizontal grid spacing of $2.8 \mathrm{~km}$ and 50 hybrid vertical layers which follow the terrain at lower levels and are horizontally flat in the upper levels. The model is continuously cycled and 
the lateral boundary conditions are provided every hour by the ICON-EU nest of the Icosahedral Nonhydrostatic global model (ICON) (Zängl et al. 2015). The ICON-EU model is run with a horizontal grid spacing of $6.5 \mathrm{~km}$ for the deterministic and $20 \mathrm{~km}$ for the ensemble run.

An ensemble Kalman filter for convective-scale data assimilation (KENDA) has been developed for the COSMO model. For a more complete description of the COSMO-KENDA system we refer the reader to Schraff et al. (2016). The KENDA system comprises a local ensemble transform Kalman filter (LETKF) that employs a square root filter to calculate the analysis ensemble from the background ensemble (Hunt et al. 2007). Observations are used both at the analysis time and during the period up to the next analysis time resulting in a 4D LETKF. As well as the analysis ensemble, the KENDA system provides a deterministic analysis. The deterministic assimilation is updated by using the Kalman gain for the ensemble mean from the LETKF, as described in Schraff et al. (2016), and the innovations (observation minus first guess) of the deterministic run.

KENDA became operational in March 2017. The ensemble consists of 40 members. In the operational setting conventional observation types (radiosondes, aircraft, wind profiler, and synoptic surface data) and Mode-S aircraft data are assimilated. An adaptive horizontal localization is applied for these observations in order to keep the number of observations more or less constant for every analysis point. To avoid deterioration of spread, the KENDA system makes use of an adaptive multiplicative covariance inflation plus relaxation to prior perturbation. An additive covariance inflation is also used in the operational system. Furthermore, soil moisture perturbations are applied to account for some model error. Radar derived rain rates, based on $2 \mathrm{D}$ reflectivity data from terrain following radar precipitation scans, are assimilated by applying latent heat nudging to each ensemble member (see Stephan et al. 2008; Schraff et al. 2016).

\section{b. Doppler radar radial winds}

Radial wind observations are obtained by measuring the phase shift between a transmitted electromagnetic wave pulse and its backscatter echo using Doppler radars. The "Doppler shift" is then used to estimate the radial velocity of the scattering target (Doviak and Zrnic 1993). In this work we consider only observations where the scattering targets are assumed to be precipitation; however, it is possible to derive clear air radar returns (e.g., Rennie et al. 2010, 2011).

The DRW data used at the DWD are acquired using a radar network of $17 \mathrm{C}$-band Doppler radars covering Germany and surrounding areas; the location of the radars

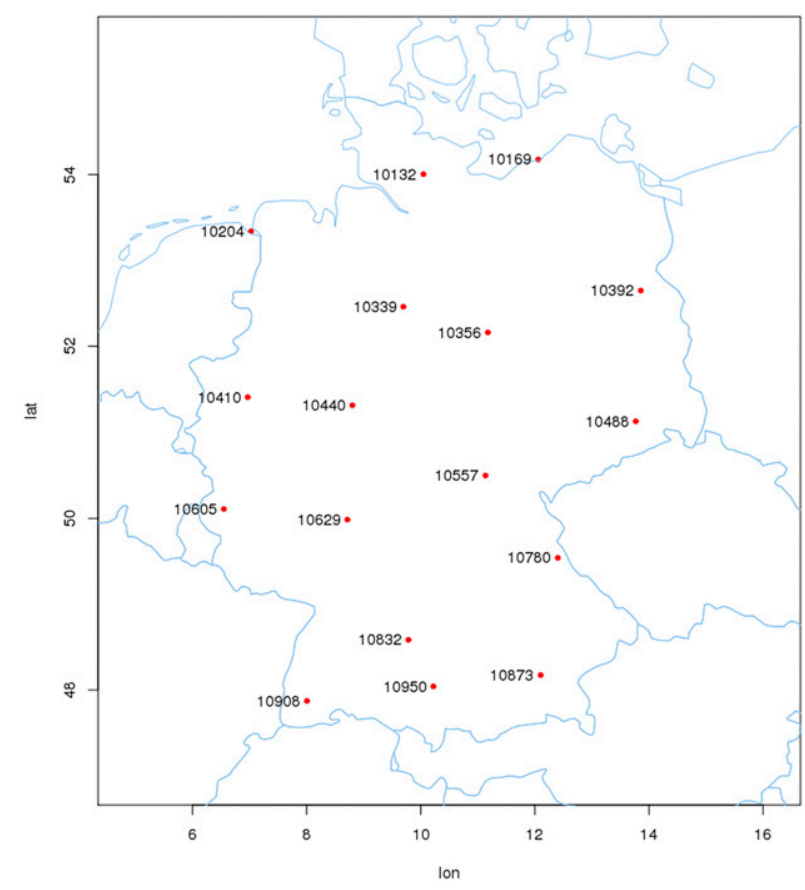

FIG. 1. Location of DWD radars.

are given in Fig. 1. Each radar completes a series of plan position indicator (PPI) scans out to a range of $180 \mathrm{~km}$ every $5 \mathrm{~min}$ at 10 different elevation angles $\left(0.5^{\circ}, 1.5^{\circ}\right.$, $2.5^{\circ}, 3.5^{\circ}, 4.5^{\circ}, 5.5^{\circ}, 8^{\circ}, 12^{\circ}, 17^{\circ}$, and $25^{\circ}$ ) with a $1^{\circ} \times 1 \mathrm{~km}$ resolution volume. The position of these elevations is shown in Fig. 2. Before assimilation the data has to pass quality control (QC) procedures. A first QC is done at the radar sites by signal processor filters (see e.g., Werner 2014, his section 2.2.). Then, during the forecast cycle, prior to assimilation and superobbing, the radar operator performs dealiasing based on simulated background wind (the folding velocity is $32 \mathrm{~m} \mathrm{~s}^{-1}$.).

Due to the resolution of the radar scan there is potential for the number of DRW observations to be very large. Close to the radar, the observation resolution is much higher than the resolution of the model. To mitigate against the large number of high density observations, several radar gates are combined into superobservations. We note that, in general, there are two main approaches to creating superobservations; one adds average innovation values to the background value at the superobservation location (e.g., Daley (1991); Simonin et al. (2014)); the other, used at DWD, simply averages observations (e.g., as in Alpert and Kumar (2007); Salonen et al. (2009); Bick et al. (2016)).

The superobbing procedure used at DWD for DRWs is the same as that described by Bick et al. (2016) employed for calculating radar reflectivity superobservations. A schematic of the procedure is given in Fig. 3. 


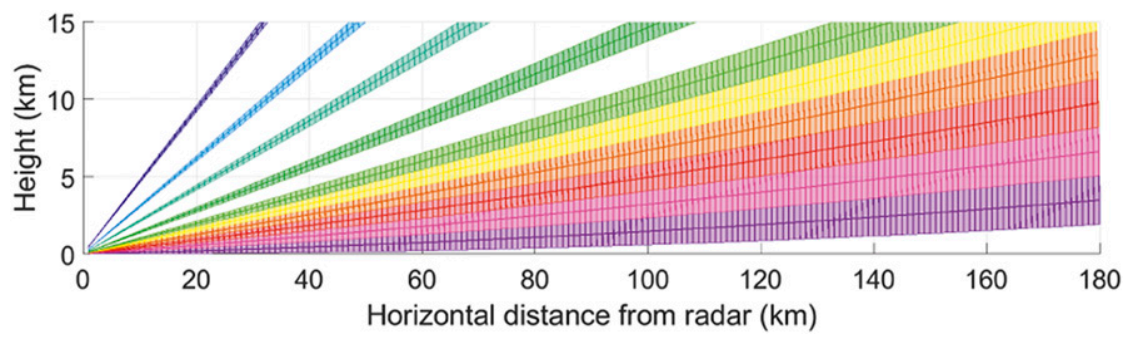

FIG. 2. A typical radar beam at elevations $0.5^{\circ}$ (purple), $1.5^{\circ}$ (pink), $2.5^{\circ}$ (red), $3.5^{\circ}$ (orange), $4.5^{\circ}$ (yellow), $5.5^{\circ}$ (light green), $8^{\circ}$ (dark green), $12^{\circ}$ (turquoise), $17^{\circ}$ (light blue), and $25^{\circ}$ (dark blue).

The superobbing procedure is applied to every PPI scan individually (i.e., to each elevation and each radar station) during the model run. The radar grid is overlaid with a Cartesian grid (blue dots in Fig. 3) with a given resolution $L_{x}$. For every Cartesian grid point the algorithm looks for the nearest radar gate (black circles) which then will become the location of the superobservation. If this point is too close to the radar station (i.e., in the experiment within a range of around $10 \mathrm{~km}$ ), it is omitted (black cross). Otherwise a wedge around this radar point is constructed with range $\pm\left(L_{x} \sqrt{2} / 2\right)$ and azimuth $\pm \arctan \left(L_{x} \sqrt{2} / 2 r_{0}\right)$ at range $r_{0}$ (green or light pink wedge in Fig. 3). The azimuthal extent can be further restricted (in this experiment at most $\pm 5^{\circ}$ ) (light brown wedge over the light pink wedge in Fig. 3). If there are at least three observations within the superobbing wedge and if their standard deviation does not exceed a threshold of $10 \mathrm{~m} \mathrm{~s}^{-1}$, the superobservation is obtained by averaging over this area. If there are fewer than three observations no superobservation is created. The number of raw observations used to calculate superobservations decreases with range (165 gates at near range, 75 at far range). We note that it is possible for the same raw observation to be used in multiple superobservations. The superobbing procedure can be understood as a transformation of the observations by a linear operator and so is not, in principle, a problem for the assimilation procedure. However, as we show in

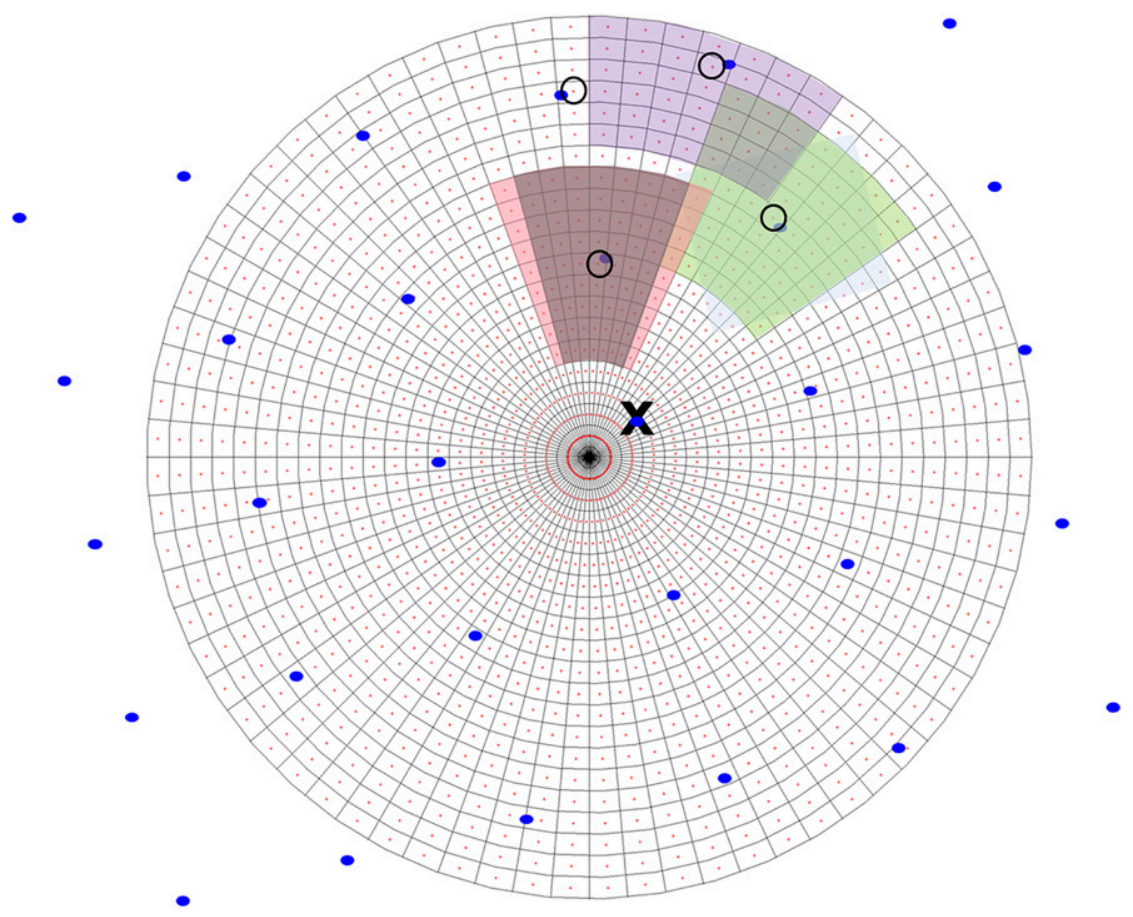

FIG. 3. Schematic of superobbing procedure. The radar grid is overlaid by a cartesian grid (blue dots). The radar gates that best fit the Cartesian grid points are the superobbing locations (black circles). The superobbing value is the average over the radar data in a surrounding wedge. Further explanation of this schematic is given in section $3 b$. 
section $5 \mathrm{a}(2)$, the use of the same raw observation in multiple superobservations causes correlation between superobservation errors.

Before the superobservations are used in the assimilation system they have to pass a first guess check. This check is based on the deterministic run and takes into account the spread of the ensemble and the observation errors. Once the observations have been quality controlled and the superobservations created they are assimilated using the COSMO-KENDA system described in section 3a. The DRWSO observation error standard deviation used in the assimilation is set to be $3 \mathrm{~m} \mathrm{~s}^{-1}$ for all elevations and all heights. For the LETKF the localization is determined by the fifth-order Gaspari-Cohn function (Gaspari and Cohn 1999) with length scales of to $16 \mathrm{~km}$ in the horizontal and $0.3 \log (\mathrm{hPa})$ in the vertical.

\section{c. Model Doppler radar radial winds}

The observations are compared to model Doppler velocities simulated using the efficient modular volume scanning radar operator (EMVORADO) (Zeng et al. 2016). First, the three-dimensional wind vector $\mathbf{v}$ is trilinearly interpolated from the model grid to the radar gates, see also (Zeng et al. (2016), Fig. 3). Then, the radial velocity $v_{r}$ is calculated as in Eq. (26) of Zeng et al. (2016):

$$
v_{r}\left(r_{0}\right)=\mathbf{v}\left(r_{0}\right) \cdot \mathbf{e}_{r}-\left(\mathbf{e}_{3} \cdot \mathbf{e}_{r}\right) \cdot \overline{w_{t}},
$$

where $\mathbf{e}_{r}$ is the unit vector in the radial direction, assuming an effective Earth radius of $4 / 3$ true Earth radius for beam bending, and $\mathbf{e}_{3}$ is the unit vector in the vertical direction. The mean value of the local terminal fall speed $\overline{w_{t}}$ is calculated as in the second part of Eq. (15) of Zeng et al. (2016) without weighting by reflectivity. It takes into account the local set of hydrometeors (cloud water, cloud ice, cloud rain, snow, graupel) and the particle size distributions. Thus, if there are no hydrometeors in the model (no rain) the fall speed is $0 \mathrm{~m} \mathrm{~s}^{-1}$. The model radial velocities are calculated for all radar gates for all PPI scans. The superobbing procedure is then applied to the model Doppler velocities to allow for direct comparison of the model superobservation to the superobservations.

\section{Experimental design}

\section{a. DRWSO error statistics estimation}

We use the diagnostic described in section 2 to estimate observation error statistics for DRWSOs assimilated into the convection-permitting model used at DWD. To calculate estimates of the observation error covariances we require background and analysis residuals.
We use background and analysis residuals from a data assimilation experiment for the period 25 May-10 June 2016. In these two weeks Germany suffered from several severe convective events, combined with low advection, that caused severe floods (e.g., Braunsbach flood 29 May 2016). The experiments use KENDA with conventional data and radial wind data with a supperobbing box of $L_{x}=10 \mathrm{~km}$, resulting in a range of $\pm 7 \mathrm{~km}$ and azimuth of $\pm \arctan \left(10 \times \sqrt{2} / 2 r_{0}\right)$ at range $r_{0}$. For the experiments performed here there were two differences in the COSMO-KENDA model compared to the operational KENDA system described in section 3a. Both the latent heat nudging and additive covariance inflation were not used in the experiments for this paper.

When considering DRWSO error statistics we note that error correlations can exist both spatially (along the beam, horizontally or vertically) and in time. Here we calculate horizontal error statistics for DRWSOs. Horizontal correlations indicate how observations at a given height are correlated [e.g., see Fig. 1 of Waller et al. (2016c)]. In our experiments we consider the horizontal correlations separately for each elevation (i.e., we consider the correlation between observations at the same height at a given elevation). The uncertainty estimation is stratified in this way to ensure that we compare observations which are anticipated to have similar representation errors. To calculate correlations, data from each radar scan is sorted into 200-m height layers. All observations that fall into a particular height layer are considered. The data are binned by separation distance for each pair of observations and from this the correlations are calculated. We calculate "average" horizontal correlations using data from all radars, and also compare variations in the error statistics between individual radar stations. When comparing correlations we consider the estimated correlation length scale. We determine the correlation length scale by considering where the correlation becomes insignificant $(<0.2)$ (Liu and Rabier 2002).

It is important to note that, due to the $\mathrm{QC}$ and superobbing procedures and the nature of DRW observations, it is likely that only part of a radar scan contains observations. Hence, the data availability differs for each elevation and each radar; this means that it is not possible to estimate standard deviations and correlations for every height at every elevation. To ensure that sampling error does not contaminate our estimates of the error statistics we discard any estimated standard deviation or correlations where there are fewer than 1000 samples used for the estimation. Furthermore, due to limitations with the diagnostic applied to LETKF analyses (discussed in section 2) it is only possible to estimate correlations out to a separation distance shorter than the observation cutoff distance determined 
TABLE 1. Differences between the experimental design in this manuscript (DWD) and Waller et al. (2016c) (Met Office).

\begin{tabular}{|c|c|c|}
\hline Variable & DWD & Met Office \\
\hline Time period of experiment & 25 May-10 Jun 2016 & 1 Jun-31 Aug 2013 \\
\hline Model domain & Germany & United Kingdom \\
\hline Model resolution & $2.8 \mathrm{~km}$ & $1.5 \mathrm{~km}$ \\
\hline Assimilation methodology & LETKF & 3D-Var \\
\hline Assimilation resolution & $8.4 \mathrm{~km}$ & $3 \mathrm{~km}$ \\
\hline Radar beam elevations & $0.5^{\circ}, 1.5^{\circ}, 2.5^{\circ}, 3.5^{\circ}, 4.5^{\circ}, 5.5^{\circ}, 8^{\circ}, 12^{\circ}, 17^{\circ}, 25^{\circ}$ & $1.0^{\circ}, 2.0^{\circ}, 4.0^{\circ}, 6.0^{\circ}$ \\
\hline Radar resolution & $1 \mathrm{~km} \times 1^{\circ}$ & $0.6 \mathrm{~km} \times 1^{\circ}$ and $0.075 \mathrm{~km} \times 1^{\circ}$ \\
\hline QC & See section $3 b$ & See Simonin et al. (2014) \\
\hline Superobbing procedure & Based on data average (Bick et al. 2016) & Based on innovation average (Simonin et al. 2014) \\
\hline $\begin{array}{l}\text { Assumed observation error } \\
\text { standard deviation }\end{array}$ & $3 \mathrm{~m} \mathrm{~s}^{-1}$ & $1.8-2.8 \mathrm{~m} \mathrm{~s}^{-1}$ \\
\hline
\end{tabular}

by the localization length scale. In our experiments the localization length scale is $16 \mathrm{~km}$, using the fifth-order Gaspari-Cohn function (Gaspari and Cohn 1999) this results in an observation cutoff distance of approximately $60 \mathrm{~km}$; therefore we estimate correlations for separation distances less than $50 \mathrm{~km}$. We also estimated the observation error statistics from analyses calculated using a larger localization radius $(32 \mathrm{~km})$ for the DRWs. We do not show the results here but note that relationship between estimated variance and height/range and the relationship between correlation length scale and height are qualitatively similar to those shown here.

As discussed in section 3a, from the assimilation we can obtain both the deterministic analysis and the analysis ensemble mean. It is possible to calculate error statistics using the analysis residuals from either the deterministic analysis or analysis ensemble mean, but we will focus on error statistics calculated using the deterministic analysis. We chose the deterministic analysis, since the ensemble mean does not necessarily give a solution consistent with the model. Error statistics estimated using the ensemble analysis mean were initially considered and showed qualitatively similar results to those given in section 5. However, it was found that the standard deviations were slightly smaller and correlation length scales slightly larger when estimated using the analysis ensemble mean. We hypothesize that this is a result of the smoothed fields in the analysis ensemble mean.

\section{b. Comparison to previous results}

In an attempt to understand the sources of observation error further we compare our estimated DRWSO error statistics for the DRWSOs that were assimilated using the COSMO-KENDA system with the DRWSO error statistics estimated in Waller et al. (2016c) for the Met Office UKV model. We note that Waller et al. (2016c) concluded that:
- DRWSO error standard deviations and correlation length scales increase with height (as a function of the increase in measurement volume).

- The DRWSOs exhibit large spatial correlations that increase with height.

- The approximate nature of the observation operator results in error correlation at all heights, with the correlation increasing with distance from the radar.

- At close range the superobbing procedure does not result in error correlation. For observations farther from the radar the superob cell size increases which results in error correlations.

For our comparison we consider DWD results from the lowest eight elevations, and for all elevations we only consider error statistics for those observations below $10 \mathrm{~km}$. When comparing our results we must bear in mind that, although the error estimation was performed using the same methodology as described in the previous section, there are a number of differences between the experimental design in this manuscript and Waller et al. (2016c). These relate to the model, assimilation and time periods of the experiment and are detailed in Table 1. The observation operators in both systems are similar, with the DRW being modeled as a point observation; the only difference is the inclusion of the terminal fall speed in the DWD observation operator. The largest difference between the DWD and Met Office observation processing is the superobbing procedure. As described in section $3 \mathrm{~b}$ the superobservations at DWD are created by averaging the observations in a given area; in contrast, at the Met office the superobservations are created by averaging the observation-minus-background values in a given area, and adding this average innovation to the background value at the superobservation location. Furthermore, the Met office procedure ensures that any raw observation can only be used in a single superobservation, whereas in the DWD methodology a raw 

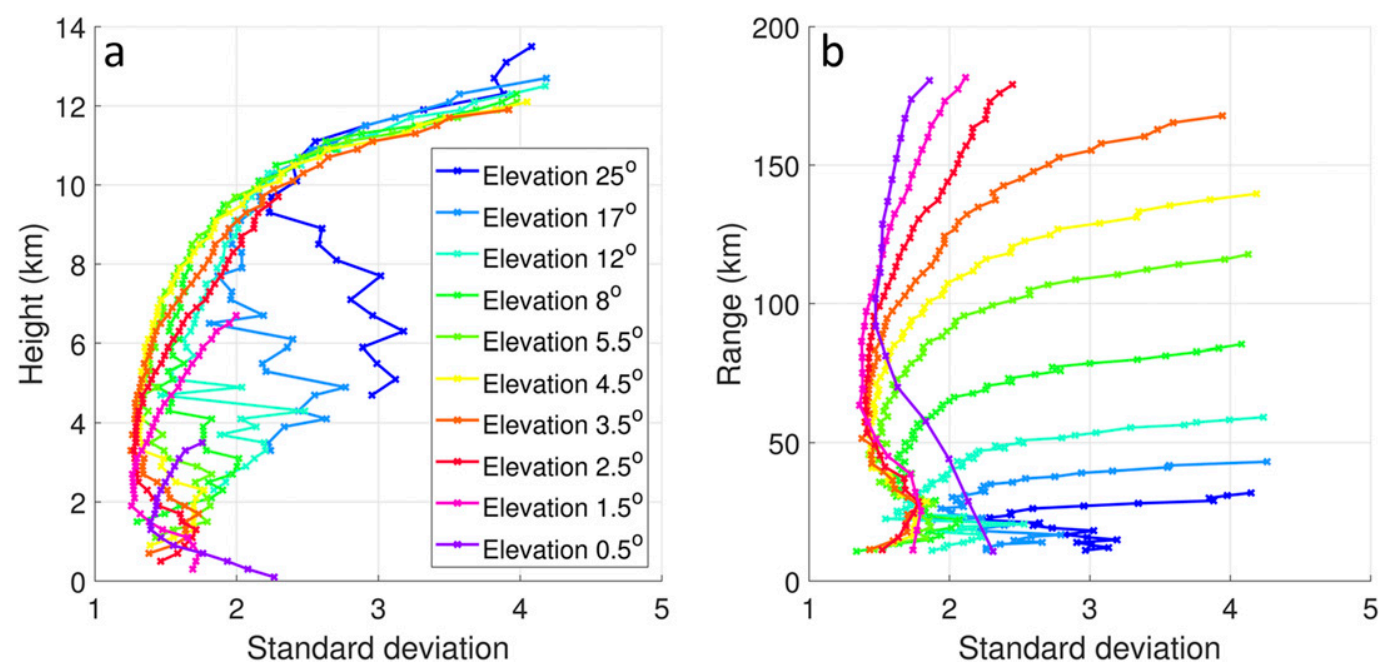

FIG. 4. Error standard deviations estimated using the deterministic analysis for $0.5^{\circ}$ (purple), $1.5^{\circ}$ (pink), $2.5^{\circ}$ (red), $3.5^{\circ}$ (orange), $4.5^{\circ}$ (yellow), $5.5^{\circ}$ (light green), $8^{\circ}$ (dark green), $12^{\circ}$ (turquoise), $17^{\circ}$ (light blue), and $25^{\circ}$ (dark blue). (a) Standard deviations plotted with observation height. (b) Standard deviations plotted with observation range from radar.

observation can be used in the creation of multiple superobservations.

\section{Results}

\section{a. Estimated observation error standard deviations and horizontal correlations for all radars}

We begin by using observations from all radar scans to calculate the observation error statistics at different heights for each elevation separately.

\section{1) STANDARD DEVIATIONS}

In Fig. 4a we plot the standard deviation profiles with height for each elevation; the same result is plotted against distance from the radar (range) in Fig. 4b. We see that in general the standard deviations range between 1 and $4.5 \mathrm{~m} \mathrm{~s}^{-1}$. The top three elevations $\left(12^{\circ}, 17^{\circ}\right.$, $25^{\circ}$ ) exhibit noisy behavior and in general the standard deviations are larger. In part, this may be a result of the reduced number of samples used for the standard deviation estimation. In addition to the large standard deviations for the high elevations, we also note that for all elevations the observation errors become large above $10 \mathrm{~km}$. The large errors in the highest elevations, and for all elevations above $10 \mathrm{~km}$ may be explained by simplifications in the observation operator. The observation operator only uses a mean hydrometeor fall speed instead of accounting for the weighting by reflectivity [see also Zeng et al. 2016, Eq. (15)]. The mean hydrometeor fall speed might differ from the actual fall speed and affects the highest elevations in particular. The large standard deviations suggest that currently it may not be beneficial to assimilate observations high in the atmosphere or from high-elevation beams. If we consider only the observations in the first 7 beams below $10 \mathrm{~km}$, the range of standard deviations is much smaller (between 1 and $2 \mathrm{~m} \mathrm{~s}^{-1}$ ). These estimated standard deviations are smaller than the standard deviation, $3 \mathrm{~m} \mathrm{~s}^{-1}$, that is used in the assimilation.

The estimated observation error standard deviation profiles for elevations $1.5^{\circ}, 2.5^{\circ}, 3.5^{\circ}, 4.5^{\circ}, 5.5^{\circ}$, and $8^{\circ}$ all exhibit similar features. Figure 4 a shows that initially the standard deviations increase with height; they reach a "low level peak" before decreasing to reach a minimum; they then increase slowly with height again. We note that the peak at low heights occurs at approximately the same range, $30 \mathrm{~km}$, for each elevation (Fig. 4b). We attribute the general increase in error variances with height to the observation operator. We note that for each elevation the volume of atmosphere sampled by the raw observation increases with height. For any given height the volume sampled by the high-elevation beam will be smaller than the low elevation beam. Observations that sample larger volumes are subject to more error from the observation operator. The effect of this can be seen in Fig. 4a where at any given height, the standard deviations are larger for lower elevations. The cause of reduction in error variance between 30 and $60 \mathrm{~km}$ is unidentified; however, additional experiments with a superobbing grid where $L_{x}=20$ (not shown) identify that the overlapping superobservation wedges were not the cause of the decrease in variances. 


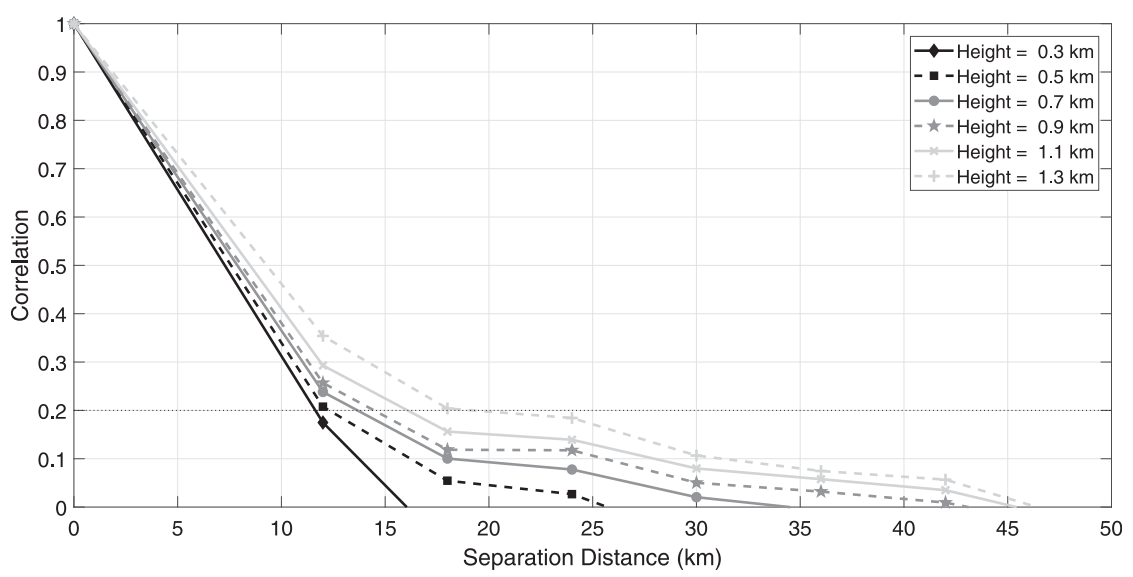

FIG. 5. Estimated correlations (deterministic analysis) for $1.5^{\circ}$ beam for heights between 0.3 and $1.3 \mathrm{~km}$.

For the lowest elevation, $0.5^{\circ}$, the estimated standard deviations decrease between the surface and $1 \mathrm{~km}$. The standard deviations then increase slightly as height increases. For this elevation the largest standard deviations occur at the lower levels and the low-level peak in the error profile is not seen. The $0.5^{\circ}$ beam is the most susceptible to clutter and large estimated standard deviations suggest that contaminated observations have been assimilated. Studying the raw radar scans shows that, despite a clutter removal step in the quality control procedure, assimilation of clutter is occurring. Currently, assimilation tests with new quality control procedures (Werner 2014) are ongoing at DWD. This result highlights that the diagnostic can also be used to understand defects in the observation processing and assimilation system.

\section{2) Correlations}

We now use observations from all radar scans to calculate the horizontal observation error correlations at different heights for each elevation separately.

We first consider the estimated horizontal correlations at different heights for the low elevation beams $\left(0.5^{\circ}, 1.5^{\circ}, 2.5^{\circ}, 3.5^{\circ}, 4.5^{\circ}\right)$. In Figs. 5 and 6 we show the correlations estimated for the $1.5^{\circ}$ beam. We note that the other low-level beams exhibit qualitatively similar behavior. In Fig. 5 we see that the lowest height has shortest correlation length scale which is approximately $11 \mathrm{~km}$. The correlation length scales then increase with height (up to a height of $1.3 \mathrm{~km}$ ) to approximately $18 \mathrm{~km}$. Figure 6 shows that above the height of $1.9 \mathrm{~km}$ the correlation length scales no longer increase with height. The correlation length scale becomes more similar for each height with length scales in the range 17 to $20 \mathrm{~km}$.

When considering the estimated correlations for the high elevations $\left(5.5^{\circ}, 8^{\circ}, 12^{\circ}, 17^{\circ}, 25^{\circ}\right)$, the correlation length scale increases with height (Fig. 7), although the relationship is not as distinct as in the low elevations at low heights. The range of correlation length scales is similar to that for the lower elevations, being between 11 and $20 \mathrm{~km}$.

It is clear that there are two different regimes for the correlation length scales. At near ranges the correlation length scale is a function of height, but at far range the correlations appear independent of height. We hypothesize that the change in regime is a result of the superobbing procedure. To investigate, we carry out an experiment using pseudosuperobservations to examine the effect of the overlapping superobbing wedges. These simple model experiments show that at far ranges there is significant overlap in the superobbing wedges, with raw observations being used to make several superobservations. The use of the same raw observation to make multiple superobseravtion results in correlation between superobservation errors. With our pseudoobservations we find that all observations above $2 \mathrm{~km}$ have correlated errors with a length scale of approximately $14 \mathrm{~km}$. Below $2 \mathrm{~km}$ the error correlations increase with height. Hence our simple model experiments confirm that the change in regime is related to the overlapping of superobbing wedges. In our simple experiments the estimated length scales are shorter than estimate for the DWD DRWSOs. Waller et al. (2016c) showed that the omission of beam broadening in the observation operator resulted in correlation length scales that increased with height. The observation operator used here also omits beam broadening, so we attribute the near-range increase in length scale as a function of height to the approximations in the observation operator. In general the correlation length scales are between 10 and $20 \mathrm{~km}$. Currently observation errors are assumed uncorrelated, but assimilated at a density where observation error correlations exist. This suggests 


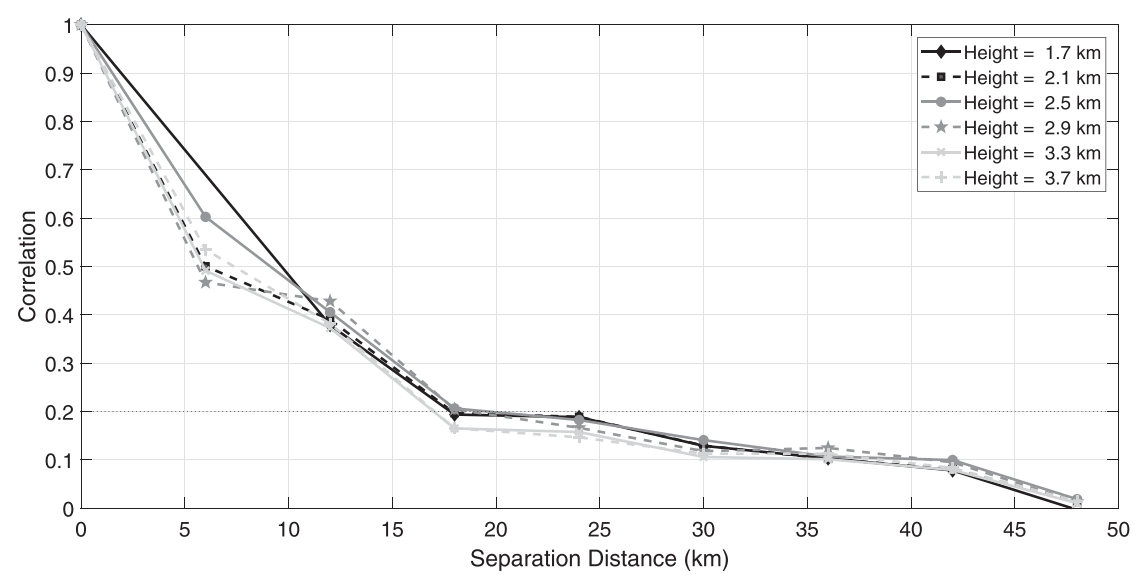

FIG. 6. Estimated correlations (deterministic analysis) for $1.5^{\circ}$ beam for heights between 1.7 and 3.7 layer.

that, if the DRWSOs are to be assimilated in their current form, either the data should be thinned or that correlations should be accounted for in the assimilation.

\section{b. Estimated standard deviations and correlations per radar}

In the previous section we combined data from all radar stations to estimate the DRWSO error statistics. Here we consider each radar individually to consider if different radars have different observation error characteristics. Calculating error statistics for each radar individually reduces the number of observations available for the estimation. We still only plot results estimated with more than 1000 samples; however, the results are not as "smooth" as those seen in the previous section.

\section{1) STANDARD DEVIATIONS}

We plot in Fig. 8 the estimated observation error standard deviations for the lowest $\left(0.5^{\circ}\right)$ elevation. The height of the radar above sea level is accounted for in the calculations. From Fig. 8 we see that the estimated standard deviations differ between radars, but each radar exhibits the same qualitative behavior as the "all radar" $0.5^{\circ}$ beam (decrease then increase with height) seen in Fig. 4b. Taking account of the radar height above sea level reduces the difference between the estimated statistics for individual radars. This suggests that standard deviations are related to the range of the observation from the radar rather than the height of the observation above the ground. We find that there are two radars that have larger error standard deviations (those with IDs 10169 and 10204). From Fig. 1 we see that these two radars take observations over the sea. Further investigation shows that the larger error standard deviations are the result of contaminated observations (contaminated by shipping lanes and wind turbines).

For the remaining elevations the individual radar standard deviations remain qualitatively similar to the

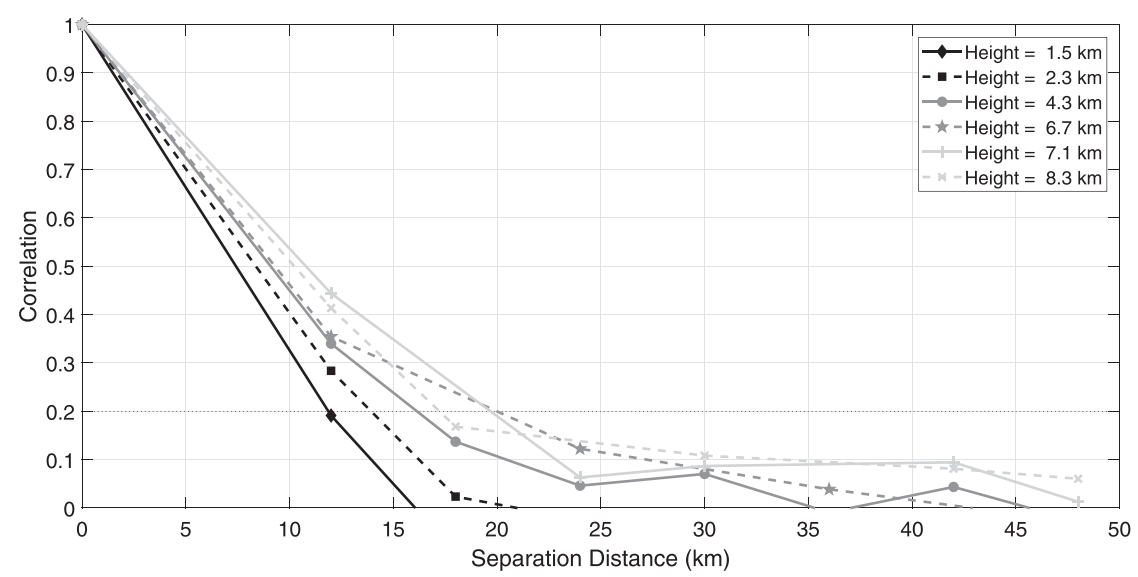

FIG. 7. Estimated correlations (deterministic analysis) for $8^{\circ}$ beam. 


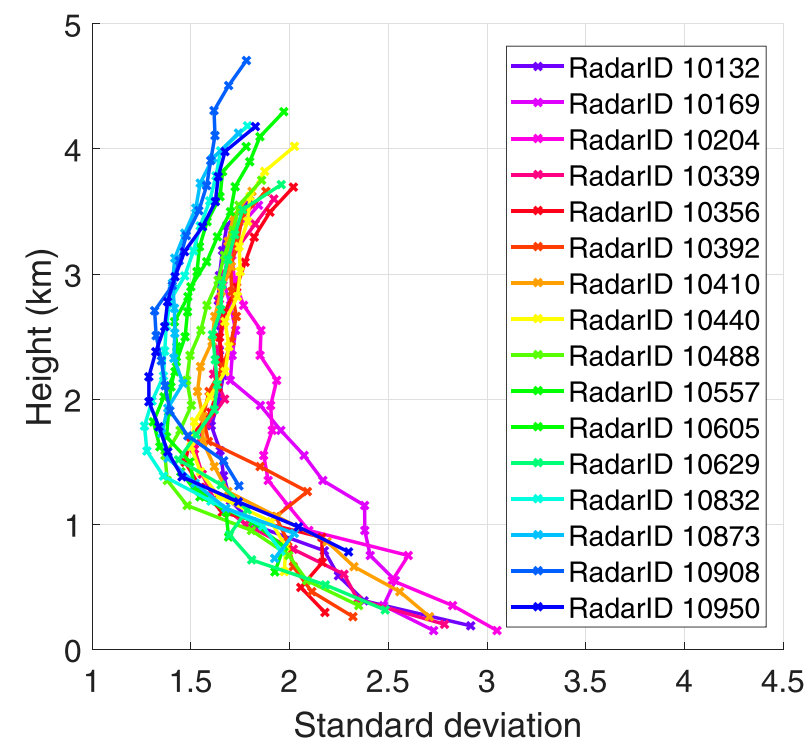

FIG. 8. Error standard deviations estimated using the deterministic analysis for $0.5^{\circ}$ beam for individual radars.

all radar standard deviations. In general the errors are more similar between radars with the exception of radar 10169.

\section{2) Correlations}

When considering the estimated correlations (not shown), again we find that the results are qualitatively similar to the all radar case. For the low elevations, initially the correlation length scales increase with height. Observations at higher heights have similar length scales. For high elevations the correlation length scales increase with height. However, we note that there are quantitative differences between individual radars.

\section{c. Comparison to previous results}

We now compare the new error statistics we have estimated for the DWD DRWSOs to the error statistics estimated for DRWs assimilated in to the Met Office system. A full description of the Met Office system can be found in section $4 \mathrm{~b}$ and Waller et al. (2016c).

\section{1) StAndard DEViations}

For both the Met Office and DWD cases we find that the standard deviations range between 1 and $2.5 \mathrm{~m} \mathrm{~s}^{-1}$. In Fig. 4a we showed that for the DWD error profiles above $4 \mathrm{~km}$ at any given height the standard deviations are larger for lower elevations. The results shown in Fig. 4a exhibit qualitatively similar behavior to the Met Office results shown in Fig. 4 of Waller et al. (2016c). We attribute this behavior to approximations in the observation operator. We note that although DWD and the Met Office use different observation operators, they both become more approximate at far range (higher heights).

For most elevations we find that the estimated standard deviations are smaller for the DWD observations. It is possible that this is a result of the diagnostic underestimating the observation error standard deviations due to the background error variance inflation required by the DWD data assimilation scheme. If the background error variance is overinflated the diagnostic will underestimate the observation error variance (Waller et al. 2016b).

The main difference in the estimated error profiles occurs at near range (low height). For the Met Office the DRW standard deviations increase with height, whereas the DWD DRW standard deviations first increase then decrease with height before increasing again. As discussed in section 4, the DWD and Met Office use very different superobbing procedures and hence we hypothesize that the differences in the low-level error variance profiles are a result of the different superobbing procedures used.

\section{2) Correlations}

From Fig. 5 of Waller et al. (2016c) and the results in section $5 \mathrm{a}(2)$, Figs. 5 and 6 , we see that the estimated correlations for the Met Office and DWD observations exhibit qualitatively similar behavior; the length scales are longer than the thinning/superobbing distances and for any given elevation the observation error correlation length scale initially increases with height. For the Met Office observations, it was found that for any given height the observation error correlation length scales decrease with elevation increase; this is not seen for the DWD DRW error correlations. For the DWD observations, as a result of the superobbing procedure [see section $5 \mathrm{a}(2)$ ] the correlation length scales no longer increase after a particular height (the exact height is dependent on the elevation). Therefore the differences in the correlation length scales at large heights are a result of the different superobbing procedures used.

\section{Conclusions}

Improved specification of observation uncertainties is required for the efficient use of high-resolution observations in convective-scale data assimilation. The aim of this paper is to estimate observation error statistics for DRWSOs assimilated into the DWD COSMO-KENDA system. We also consider the impact of different observation processing and data assimilation schemes by comparing our results to those found in previous studies. 
Our results show that the observation error standard deviation profiles are influenced by the effects of the observation operator, which becomes less accurate with height, and by the superobbing procedure. For observations below $10 \mathrm{~km}$, the estimated observation standard deviations are lower than currently assumed in the DWD KENDA system. This suggests that it may be feasible to reduce the DRWSO error standard deviations in the assimilation scheme. The exception is the lowest observations from the lowest elevation; these observations have large error standard deviations due to the assimilation of observations contaminated by shipping lanes and wind turbines. This highlights the use of the diagnostic for determining shortcomings in the observation processing system. Since carrying out this study new quality control routines are applied to radar data within the DWD (see e.g., Werner 2014). In contrast to the low-level observations, observations above $10 \mathrm{~km}$ and those from high-elevation radar beams have much larger standard deviations and the error profiles exhibit noisy behavior. This may be a result of omissions in the observation operator or reduced sample sizes. These large standard deviations suggest that the observations high in the atmosphere and from high beams are subject to a large representation error and may not be as beneficial for assimilation.

For all elevations we find that initially the correlation length scales increase with height; we hypothesize that this is a result of the omission of beam broadening in the observation operator. For the low elevations after a given height the trend in correlations stops and the length scales at higher heights fall within a given range. This is likely a result of the superobbing procedure. For the estimated correlations we find that in general the correlation length scale is $12 \mathrm{~km}$ or longer; hence, the current assimilation incorrectly assumes that the DRWSO errors are uncorrelated.

When comparing our results to those of Waller et al. (2016c), who calculated error statistics for DRW observations used at the Met Office, we find a number of similarities. The increase in error standard deviation with height (at far ranges) and the increase in correlation length scale with measurement volume occur for both the DWD and Met Office DRW statistics. Both these features are a result of the approximate observation operator. The approximations are reasonable near the radar, but become poorer as observation distance from the radar increases and hence measurement volume, increases. The main difference between the results is the standard deviation profile at low heights which can be attributed to the different superobbing procedures. In general, the results found using the DWD ensemble system are similar to the results found in using the Met
Office variational system. The consistency between results provides further confidence in the results that can be obtained from the diagnostic, despite its limitations.

The results shown here provide an understanding of DRWSO error statistics. We are able to determine some of the main contributors, the observation operator and superobbing procedure, to the error standard deviation and correlations. Some of the error sources can be easily removed by improving the quality control procedures. Previously it has been shown by Waller et al. (2016c) that it is possible to reduce the error correlation by using an improved observation operator. Therefore, the use of the more complex version of the observation operator may be beneficial. It may also be possible to reduce the error correlation length scales by modifying the superobbing scheme (e.g., by forming the superobservations on a coarser grid ensuring that a raw observation is only used once). However, even if some of the errors can be reduced, it is likely that significant correlation will still remain for some heights at some elevations. Hence, for the improved assimilation of DRW observations it will be important to account for the correlated observation errors in the data assimilation system.

Acknowledgments. J. A. Waller, S. L. Dance, and N. K. Nichols were supported in part by U.K. NERC Grants NE/K008900/1 (FRANC) and NE/N006682/1 (OSCA). S. L. Dance and J. A. Waller received additional support from U.K. EPSRC Grant EP/P002331/1 (DARE). N. K. Nichols was also supported by the U.K. NERC National Centre for Earth Observation (NCEO). E. Bauernschubert has been supported by the research program innovation in applied research and development (IAFE) funded by the German Ministry for Transport and Digital Infrastructure (BMVI). We thank U. Blahak and K. Stephan from DWD for their help and fruitful discussions. The data used in this study may be obtained on request, subject to licensing conditions, by contacting the corresponding author.

\section{REFERENCES}

Alpert, J. C., and V. K. Kumar, 2007: Radial wind super-obs from the WSR-88D radars in the NCEP operational assimilation system. Mon. Wea. Rev., 135, 1090-1109, https://doi.org/ 10.1175/MWR3324.1.

Baldauf, M., A. Seifert, J. Förstner, D. Majewski, M. Raschendorfer, and T. Reinhardt, 2011: Operational convective-scale numerical weather prediction with the COSMO model: Description and sensitivities. Mon. Wea. Rev., 139, 3887-3905, https://oi.org/ 10.1175/MWR-D-10-05013.1.

Ballard, S. P., Z. Li, D. Simonin, and J.-F. Caron, 2016: Performance of 4D-Var NWP-based nowcasting of precipitation at the Met Office for summer 2012. Quart. J. Roy. Meteor. Soc., 142, 472-487, https://doi.org/10.1002/qj.2665.

Bennitt, G. V., H. R. Johnson, P. P. Weston, J. Jones, and E. Pottiaux, 2017: An assessment of ground-based GNSS 
zenith total delay observation errors and their correlations using the Met Office UKV model. Quart. J. Roy. Meteor. Soc., 143, 2436-2447, https://doi.org/10.1002/qj.3097.

Bick, T., and Coauthors, 2016: Assimilation of 3D radar reflectivities with an ensemble Kalman filter on the convective scale. Quart. J. Roy. Meteor. Soc., 142, 1490-1504, https://doi.org/10.1002/ qj. 2751.

Bormann, N., M. Bonavita, R. Dragani, R. Eresmaa, M. Matricardi, and A. McNally, 2016: Enhancing the impact of IASI observations through an updated observation-error covariance matrix. Quart. J. Roy. Meteor. Soc., 142, 1767-1780, https://doi.org/ 10.1002/qj.2774.

Campbell, W. F., E. A. Satterfield, B. Ruston, and N. L. Baker, 2017: Accounting for correlated observation error in a dualformulation $4 \mathrm{~d}$ variational data assimilation system. Mon. Wea. Rev., 145, 1019-1032, https://doi.org/10.1175/MWR-D16-0240.1.

Chung, K.-S., W. Chang, L. Fillion, and M. Tanguay, 2013: Examination of situation-dependent background error covariances at the convective scale in the context of the ensemble Kalman filter. Mon. Wea. Rev., 141, 3369-3387, https://doi.org/ 10.1175/MWR-D-12-00353.1.

Clark, P., N. Roberts, H. Lean, S. Ballard, and C. Charlton-Perez, 2016: Convection-permitting models: A step-change in rainfall forecasting. Meteor. Appl., 23, 165-181, https://doi.org/ 10.1002/met.1538.

Cordoba, M., S. Dance, G. Kelly, N. Nichols, and J. Waller, 2017: Diagnosing atmospheric motion vector observation errors for an operational high resolution data assimilation system. Quart. J. Roy. Meteor. Soc., 143, 333-341, https://doi.org/ 10.1002/qj.2925.

Daley, R., 1991: Atmospheric Data Analysis. Cambridge University Press, $457 \mathrm{pp}$.

Desroziers, G., L. Berre, B. Chapnik, and P. Poli, 2005: Diagnosis of observation, background and analysis-error statistics in observation space. Quart. J. Roy. Meteor. Soc., 131, 3385-3396, https://doi.org/10.1256/qj.05.108.

Doviak, R. J., and D. S. Zrnić, 1993: Doppler Radar and Weather Observations. 2nd ed. Academic Press, 562 pp.

Fowler, A. M., S. L. Dance, and J. A. Waller, 2018: On the interaction of observation and prior error correlations in data assimilation. Quart. J. Roy. Meteor. Soc., 144, 48-62, https:// doi.org/10.1002/qj.3183.

Gaspari, G., and S. E. Cohn, 1999: Construction of correlation functions in two and three dimensions. Quart. J. Roy. Meteor. Soc., 125, 723-757, https://doi.org/10.1002/qj.49712555417.

Gorin, V. E., and M. D. Tsyrulnikov, 2011: Estimation of multivariate observation-error statistics for AMSU-A data. Mon. Wea. Rev., 139, 3765-3780, https://doi.org/10.1175/2011MWR3554.1.

Gustafsson, N., and Coauthors, 2018: Survey of data assimilation methods for convective-scale numerical weather prediction at operational centres. Quart. J. Roy. Meteor. Soc., 144, 1218-1256, https://doi.org/10.1002/qj.3179.

Hodyss, D., and N. K. Nichols, 2015: The error of representation: Basic understanding. Tellus, 67A, 24822, https://doi.org/10.3402/ tellusa.v67.24822.

Hunt, B. R., E. J. Kostelich, and I. Szunyogh, 2007: Efficient data assimilation for spatiotemporal chaos: A local ensemble transform Kalman filter. Physica D, 230, 112-126, https:// doi.org/10.1016/j.physd.2006.11.008.

Janjić, T., and Coauthors, 2018: On the representation error in data assimilation. Quart. J. Roy. Meteor. Soc., 144, 1257-1278, https://doi.org/10.1002/qj.3130.
Liu, Z.-Q., and F. Rabier, 2002: The interaction between model resolution observation resolution and observation density in data assimilation: A one dimensional study. Quart. J. Roy. Meteor. Soc., 128, 1367-1386, https://doi.org/10.1256/ 003590002320373337.

Lorenc, A. C., 1981: A global three-dimensional multivariate statistical interpolation scheme. Mon. Wea. Rev., 109, 701-721, https://doi.org/10.1175/1520-0493(1981)109<0701:AGTDMS>2.0.CO;2.

Ménard, R., 2016: Error covariance estimation methods based on analysis residuals: Theoretical foundation and convergence properties derived from simplified observation networks. Quart. J. Roy. Meteor. Soc., 142, 257-273, https://doi.org/ 10.1002/qj.2650.

Montmerle, T., and C. Faccani, 2009: Mesoscale assimilation of radial velocities from Doppler radars in a preoperational framework. Mon. Wea. Rev., 137, 1939-1953, https://doi.org/ 10.1175/2008MWR2725.1.

Müller, M., and Coauthors, 2017: AROME-MetCoOp: A Nordic convective-scale operational weather prediction model. Wea. Forecasting, 32, 609-627, https://doi.org/10.1175/WAF-D-160099.1.

Rennie, S. J., A. J. Illingworth, S. L. Dance, and S. P. Ballard, 2010: The accuracy of Doppler radar wind retrievals using insects as targets. Meteor. Appl., 17, 419-432, https://doi.org/10.1002/ met.174.

- , S. L. Dance, A. J. Illingworth, S. P. Ballard, and D. Simonin, 2011: 3D-Var assimilation of insect-derived Doppler radar radial winds in convective cases using a high-resolution model. Mon. Wea. Rev., 139, 1148-1163, https://doi.org/10.1175/ 2010MWR3482.1.

Salonen, K., H. Järvinen, G. Haase, S. Niemela, and R. Eresmaa, 2009: Doppler radar radial winds in HIRLAM. Part II: Optimizing the super-observation processing. Tellus, 61A, 288-295, https://doi.org/10.1111/j.1600-0870.2008.00381.x.

Schraff, C., H. Reich, A. Rhodin, A. Schomburg, K. Stephan, A. Periáñez, and R. Potthast, 2016: Kilometre-scale ensemble data assimilation for the COSMO model (KENDA). Quart. J. Roy. Meteor. Soc., 142, 1453-1472, https://doi.org/10.1002/ qj.2748.

Simonin, D., S. P. Ballard, and Z. Li, 2014: Doppler radar radial wind assimilation using an hourly cycling 3D-Var with a $1.5 \mathrm{~km}$ resolution version of the Met Office Unified Model for nowcasting. Quart. J. Roy. Meteor. Soc., 140, 2298-2314, https://doi.org/10.1002/qj.2298.

, J. A. Waller, S. P. Ballard, S. L. Dance, and N. K. Nichols, 2019: A pragmatic strategy for implementing spatially correlated observation errors in an operational system: An application to Doppler radial winds. Quart. J. Roy. Meteor. Soc., https://doi.org/10.1002/qj.3592, in press.

Stephan, K., S. Klink, and C. Schraff, 2008: Assimilation of radarderived rain rates into the convective-scale model COSMO-DE at DWD. Quart. J. Roy. Meteor. Soc., 134, 1315-1326, https:// doi.org/10.1002/qj.269.

Stewart, L. M., S. L. Dance, and N. K. Nichols, 2008: Correlated observation errors in data assimilation. Int. J. Numer. Methods Fluids, 56, 1521-1527, https://doi.org/10.1002/fld.1636.

$\longrightarrow,-$, and 2013: Data assimilation with correlated observation errors: experiments with a 1-D shallow water model. Tellus, 65A, 19546, https://doi.org/10.3402/tellusa.v65i0.19546.

,,,--- J. R. Eyre, and J. Cameron, 2014: Estimating interchannel observation-error correlations for IASI radiance data in the Met Office system. Quart. J. Roy. Meteor. Soc., 140, 1236-1244, https://doi.org/10.1002/qj.2211. 
Sun, J., and Coauthors, 2014: Use of NWP for nowcasting convective precipitation: Recent progresses and challenges. Bull. Amer. Meteor. Soc., 95, 409-426, https://doi.org/10.1175/ BAMS-D-11-00263.1.

Tang, Y., H. W. Lean, and J. Bornemann, 2013: The benefits of the Met Office variable resolution NWP model for forecasting convection. Meteor. Appl., 20, 417-426, https://doi.org/10.1002/ met.1300.

Terasaki, K., and T. Miyoshi, 2014: Data assimilation with errorcorrelated and non-orthogonal observations: Experiments with the Lorenz-96 model. SOLA, 10, 210-213.

Todling, R., 2015: A complementary note to 'A lag-1 smoother approach to system-error estimation': The intrinsic limitations of residual diagnostics. Quart. J. Roy. Meteor. Soc., 141, 2917-2922, https://doi.org/10.1002/qj.2546.

Waller, J. A., S. L. Dance, A. S. Lawless, N. K. Nichols, and J. R. Eyre, 2014: Representativity error for temperature and humidity using the Met Office high-resolution model. Quart. J. Roy. Meteor. Soc., 140, 1189-1197, https://doi.org/10.1002/ qj.2207.

S. P. Ballard, S. L. Dance, G. Kelly, N. K. Nichols, and D. Simonin, 2016a: Diagnosing horizontal and inter-channel observation error correlations for SEVIRI observations using observation-minus-background and observation-minus-analysis statistics. Remote Sens., 8, 581, https://doi.org/10.3390/rs8070581.

—, S. L. Dance, and N. K. Nichols, 2016b: Theoretical insight into diagnosing observation error correlations using observationminus-background and observation-minus-analysis statistics. Quart. J. Roy. Meteor. Soc., 142, 418-431, https://doi.org/ 10.1002/qj.2661.

— D. Simonin, S. L. Dance, N. K. Nichols, and S. P. Ballard, 2016c: Diagnosing observation error correlations for Doppler radar radial winds in the Met Office UKV model using observation-minus-background and observation-minusanalysis statistics. Mon. Wea. Rev., 144, 3533-3551, https://doi.org/ 10.1175/MWR-D-15-0340.1.

— S. L. Dance, and N. K. Nichols, 2017: On diagnosing observation-error statistics with local ensemble data assimilation. Quart. J. Roy. Meteor. Soc., 143, 2677-2686, https:// doi.org/10.1002/qj.3117.

J. García-Pintado, D. C. Mason, S. L. Dance, and N. K. Nichols, 2018: Technical note: Assessment of observation quality for data assimilation in flood models. Hydrol. Earth Syst. Sci., 22, 3983-3992, https://doi.org/10.5194/hess-22-39832018.

Wattrelot, E., T. Montmerle, and C. G. Guerrero, 2012: Evolution of the assimilation of radar data in the AROME model at convective scale. Proc. Seventh European Conf. on Radar in Meteorology and Hydrology (ERAD 2012), Toulouse, France, Météo-France, http://www.meteo.fr/cic/meetings/2012/ERAD/ extended_abs/NWP_401_ext_abs.pdf.

Werner, M., 2014: A new radar data post-processing quality control workflow for the DWD weather radar network. Proc. Eighth European Conf. on Radar in Meteorology and Hydrology, Garmisch-Partenkirchen, Germany, DWD, http://www.pa.op.dlr.de/ erad2014/programme/ExtendedAbstracts/079_Werner.pdf.

Weston, P. P., W. Bell, and J. R. Eyre, 2014: Accounting for correlated error in the assimilation of high-resolution sounder data. Quart. J. Roy. Meteor. Soc., 140, 2420-2429, https:// doi.org/10.1002/qj.2306.

Xiao, Q., and Coauthors, 2008: Doppler radar data assimilation in KMAs operational forecasting. Bull. Amer. Meteor. Soc., 89, 39-43, https://doi.org/10.1175/BAMS-89-1-39.

Xue, M., F. Kong, K. Thomas, J. Gao, Y. Wang, K. Brewster, and K. Droegemeier, 2013: Prediction of convective storms at convection-resolving 1-km resolution over continental United States with radar data assimilation: An example case of 26 May 2008 and precipitation forecasts from spring 2009. Adv. Meteor., 2013, 259052, https://doi.org/10.1155/2013/ 259052.

- M. Hu, and A. Schenkman, 2014: Numerical prediction of 8 May 2003 Oklahoma City tornadic supercell and embedded tornado using ARPS with assimilation of WSR-88D radar data. Wea. Forecasting, 29, 39-62, https://doi.org/10.1175/ WAF-D-13-00029.1.

Zängl, G., D. Reinert, P. Rípodas, and M. Baldauf, 2015: The ICON (ICOsahedral Non-hydrostatic) modelling framework of DWD and MPI-M: Description of the non-hydrostatic dynamical core. Quart. J. Roy. Meteor. Soc., 141, 563-579, https:// doi.org/10.1002/qj.2378.

Zeng, Y., U. Blahak, and D. Jerger, 2016: An efficient modular volume-scanning radar forward operator for nwp models: description and coupling to the COSMO model. Quart. J. Roy. Meteor. Soc., 142, 3234-3256, https://doi.org/10.1002/qj.2904. 